\title{
PROCESSAMENTO AUDITIVO EM IDOSOS: IMPLICAÇÕES E SOLUÇÕES
}

\section{Auditory processing in elderly: implications and solutions}

\author{
Leonardo Henrique Buss (1), Lílian Seligman Graciolli ${ }^{(2)}$, Angela Garcia Rossi ${ }^{(3)}$
}

\begin{abstract}
RESUMO
Tema: processamento auditivo em idosos. Objetivo: estudar, através de uma revisão teórica, o processamento auditivo em idosos, as desordens que o envelhecimento auditivo causam, bem como os recursos para reduzir as defasagens nas habilidades auditivas envolvidas no processamento auditivo. Conclusão: vários são os desajustes ocasionados pela desordem do processamento auditivo em idosos. É necessária a continuidade de estudos científicos nessa área para aplicar adequadas medidas intervencionistas, a fim de garantir a reabilitação do indivíduo a tempo de minimizar os efeitos da desordem auditiva sobre o mesmo.
\end{abstract}

DESCRITORES: Audição; Idoso; Percepção Auditiva; Estimulação Acústica

\section{INTRODUÇÃO}

A audição é um ato fundamental para o ser humano ao longo da vida, sendo primordial para o contato com nossos semelhantes e com a natureza, e é através dela que o ser humano torna-se capaz de exercer a comunicação ${ }^{1}$.

Atualmente mais de 15 milhões de brasileiros têm problemas de audição, e apenas $40 \%$ dos afetados reconhecem a doença. Dentre esses pacientes encontram-se os idosos, aos quais destaca-se atenção especial neste trabalho, pois o aumento da longevidade e a redução das taxas de mortalidade ocorridas nas últimas décadas do século passado mudaram o perfil demográfico do Brasil - estimativas afirmam que até o ano de 2020, espera-se alcançar um total de 32 milhões de pessoas com mais de 60 anos ${ }^{2}$, fazendo com que o envelhecimento populacional seja um dos maiores desafios da saúde pública contemporânea.

(1) Fonoaudiólogo clínico; Mestrando em Distúrbios da Comunicação Humana da Universidade Federal de Santa Maria.

(2) Fonoaudióloga; Professora Doutora do Departamento de Fonoaudiologia da Universidade Federal de Santa Maria, UFSM, Santa Maria, RS; Doutora em Engenharia de Produção pela Universidade Federal de Santa Catarina.

(3) Fonoaudióloga; Professora Doutora do Departamento de Fonoaudiologia da Universidade Federal de Santa Maria, UFSM, Santa Maria, RS; Doutora em Distúrbios da Comunicação Humana pela Universidade Federal de São Paulo.

Conflito de interesses: inexistente
Até alguns anos atrás, a preocupação em relação à audição, por parte do profissional fonoaudiólogo, era a de saber se o paciente apresentava ou não uma deficiência auditiva, ou seja, a atenção estava voltada para a questão da acuidade auditiva em termos periféricos.

O interesse na relação entre o envelhecimento e o processamento auditivo tem sido crescente, nos últimos anos, principalmente na audiologia, por causa da existência de idosos que possuem integridade auditiva periférica e/ou idosos protetizados com ganho funcional do aparelho auditivo adequado para a perda, mas que apresentam manifestações audiológicas incompatíveis com tais características audiológicas. Estudos recentes têm evidenciado que tais dificuldades podem estar relacionadas a perdas da capacidade de realizar o processamento auditivo dos sons, associadas ao envelhecimento ${ }^{3}$.

Verifica-se assim a relevância do tema para a Fonoaudiologia, a validade de estudar os efeitos da idade no processamento auditivo, além de relatar propostas terapêuticas para a redução dos danos dessa desordem auditiva, buscando assim contribuir para a saúde auditiva desta população.

Os objetivos desta recopilação são: estudar o processamento auditivo em idosos, as desordens que o envelhecimento auditivo causam, bem como recursos para reduzir as defasagens nas habilidades auditivas envolvidas neste processamento auditivo, e compartilhar as informações obtidas com os profissionais que atuam com idosos, visando propor- 
cionar melhor qualidade de vida a estes e reduzir os efeitos da desordem auditiva nesta população.

\section{MÉTODOS}

O estudo foi realizado a partir de consulta crítica e sistemática da literatura científica. Foram selecionados artigos científicos nacionais e internacionais, livros e documentos eletrônicos considerando-se a relevância e o valor informativo de tais materiais para o assunto em estudo.

Foram consultados livros científicos da área da Fonoaudiologia, sites oficiais de entidades vinculadas à audiologia e periódicos das áreas de Fonoaudiologia e Geriatria da base de dados Medline, Scirus, Pubmed, SciELO, Bireme, Amedeo e portal CAPES.

Esta recopilação foi apresentada de forma teórica organizada cronologicamente e pareada por assuntos.

\section{REVISÃO DA LITERATURA}

Neste capítulo, apresenta-se uma síntese de pesquisas compulsadas na literatura especializada sobre o processamento auditivo, as desordens que o envelhecimento auditivo causam, e as intervenções terapêuticas fonoaudiológicas. Para um melhor desenvolvimento desta revisão, optou-se pela divisão em quatro subcapítulos: I) Fisiologia da audição, II) Processamento auditivo, III) As desordens causadas pelo envelhecimento auditivo, IV) intervenções terapêuticas fonoaudiológicas relacionadas às desordens do processamento auditivo.

\section{I) Fisiologia da audição}

Um dos pré-requisitos básicos para estudar processamento auditivo central é o conhecimento morfo-funcional do sistema auditivo ${ }^{4}$.

Os componentes do sistema auditivo são três: condutivo (composto pela orelha externa e média), sensorial (cóclea) que transforma o impulso sonoro em elétrico, e neural. Os dois primeiros componentes, condutivo e sensorial, fazem parte do sistema periférico, e o neural, responsável pela atividade central, faz parte do sistema nervoso.

A atividade periférica tem a função de detecção e transmissão dos sons, e a atividade central tem a função de discriminação, localização, reconhecimento do som, compreensão, atenção seletiva e memória auditiva.

Além do sistema auditivo periférico e do sistema auditivo central, outras áreas centrais (não-auditivas) podem estar envolvidas no processamento auditivo. São elas: lobo frontal, conexão temporal- parietal, lobo occipital. Essas estruturas fazem a integração das informações sensoriais auditivas com outras não-auditivas.

\section{II) Processamento auditivo}

O processamento auditivo envolve mecanismos e processos do sistema auditivo responsáveis pela: lateralização e localização do som, discriminação auditiva, reconhecimento de padrões e aspectos temporais da audição, incluindo resolução, mascaramento, integração e ordenação temporais, desempenho auditivo com sinais acústicos competitivos e degradados ${ }^{5}$.

Processamento auditivo refere-se a uma série de processos que se sucedem no tempo e que permitem que um indivíduo realize uma análise metacognitiva dos efeitos sonoros - compreensão da fala. Envolve predominantemente as estruturas do sistema nervoso central: vias auditivas e córtex, e os processos que são classificados como detecção, sensação, discriminação, localização, reconhecimento, compreensão, memória e atenção seletiva ${ }^{6}$.

Também é definido como sendo o termo usado para descrever o que acontece quando o cérebro reconhece e interpreta os sons em torno de uma pessoa. Os seres humanos ouvem quando a energia reconhecida como som passa pela cóclea e é transformada em informação elétrica, podendo, dessa forma, ser interpretada pelo cérebro ${ }^{7}$.

Processamento Auditivo é um conjunto de habilidades específicas das quais o indivíduo depende para compreender o que ouve. É uma atividade mental, ou seja, uma função cerebral ${ }^{8}$.

O som, após ser detectado pela orelha interna, sofre inúmeros processos fisiológicos e cognitivos para que seja decodificado e compreendido ${ }^{9}$. Os comportamentos auditivos observáveis podem ser identificados como localização, discriminação auditiva, reconhecimento de padrão temporal, ordenação temporal, resolução temporal, escuta dicótica e desempenho com sinais acústicos degradados (escuta monótica). A maneira como o sistema auditivo recebe, analisa e organiza aquilo que ouvimos é chamado de processamento auditivo ${ }^{10}$.

\section{III) Desordens causadas pelo envelhecimento auditivo}

Atualmente na literatura nacional e internacional são encontrados estudos que analisam o perfil audiológico de indivíduos idosos, no que se refere às modificações na configuração da curva audiométrica, entretanto, os efeitos do processo de enveIhecimento especificamente no processamento auditivo ainda não foram documentados detalhadamente como os efeitos da idade nas alterações dos limiares auditivos ${ }^{11}$. 
A Desordem de Processamento Auditivo Central (DPAC) é a quebra em uma ou mais etapas do processamento auditivo, gerando um distúrbio de audição, em que há um impedimento na habilidade de analisar ou interpretar padrões sonoros, podendo ser decorrentes de privações sensoriais, perdas auditivas, problemas neurológicos ou outros ${ }^{12}$.

O distúrbio do processamento auditivo (DPA) refere-se à dificuldade no processamento perceptual da informação no sistema nervoso central demonstrado por um baixo desempenho em uma ou mais habilidades auditivas.

As possíveis relações entre as mudanças no processamento auditivo e o envelhecimento do sistema auditivo foram investigadas, por meio de um estudo contendo 12 idosos com idade entre 65 e 75 anos com audição normal bilateral. Sendo assim, foi enfatizada a importância de selecionar uma amostra de idosos com audição normal bilateral quando se está investigando os efeitos do processo de envelhecimento no processamento auditivo, pois, desta forma, qualquer mudança nas habilidades investigadas podem ser mais fortemente atribuídas aos fatores associados ao envelhecimento. Os resultados mostraram que o fato de sujeitos mais velhos, com limiares auditivos normais apresentarem resultados anormais e performance mais pobre que sujeitos jovens normo-ouvintes implicaria em alterações relacionadas com a idade ${ }^{13}$.

O envelhecimento é a somatória de muitos fenômenos que ocorrem após a maturação sexual em qualquer ser vivo. Muitas variáveis fazem parte do processo, como o meio ambiente, exposição a produtos tóxicos, presença de lesões físicas acidentais, nutrição do indivíduo e sua programação genética. As mudanças que não aumentam diretamente nossa vulnerabilidade para morte são chamadas de envelhecimento primário. Alguns exemplos são: embranquecimento dos cabelos, enrugamento da pele, presbiopia, perda de cabelos e redução da memória de curto prazo. Já aquelas mudanças que nos deixam mais susceptíveis, como o aumento do colesterol, diabetes e hipertensão são chamadas de envelhecimento secundário. $O$ envelhecimento envolve um aumento da susceptibilidade a agressões físicas, psicológicas e socioculturais. O sistema sensorial torna-se menos acurado, o padrão de sono muda e há declínio em alguns aspectos da memória. Há muitas deficiências sensoriais que acompanham a velhice. Elas podem levar a um isolamento social e a conseqüente desativação da vida produtiva ${ }^{14}$.

A audição e, consequentemente, a comunicação, envolvem mecanismos centrais de compreensão, processamento da informação, elaboração de uma resposta ao estímulo recebido e a emissão da mesma. Em cada etapa deste complexo processo, há perda de características do funcionamento com a idade. Levando em conta que das dez doenças crônicas mais comuns do paciente geriátrico, os problemas auditivos ocupam o quarto lugar em frequência ${ }^{15}$.

O transtorno do processamento auditivo é quando algum fator afeta de forma adversa o processamento ou interpretação de uma dada informação ${ }^{7}$.

A alteração do processamento auditivo se refere à dificuldade no processo de percepção da informação auditiva no sistema nervoso central, levando a um fraco desempenho em uma ou mais habilidades do processamento auditivo ${ }^{9}$.

O transtorno do processamento auditivo é um grupo complexo e heterogênio de alterações usualmente associada a uma série de dificuldades auditivas e de aprendizado ${ }^{16}$.

O transtorno do processamento auditivo ocorre quando existe uma representação neurofisiológica ineficiente do sinal acústico, processamento temporal e sincronia neural imprecisa, assimetria hemisférica atípica de representação auditiva (principalmente da fala) e transferência inter-hemisférica ineficiente da informação auditiva ${ }^{10}$.

\section{IV) Intervenções terapêuticas relacionadas às desordens do Processamento Auditivo}

O termo treinamento auditivo refere-se a um conjunto de condições acústicas e/ou tarefas que são indicadas para ativar a audição e sistemas relacionados de tal forma que sua base neural e os comportamentos auditivos associados sejam modificados, gerando melhora no desempenho auditivo 17. Um dos fundamentos do treinamento auditivo é a plasticidade do sistema nervoso central, definida como a mudança em células nervosas que ocorrem de acordo com as influências ambientais, sendo que estas influências podem ser controladas e modeladas de maneira desejada, ou seja, o comportamento pode estar relacionado à plasticidade previsível. Deste modo, o treinamento pode servir como influência ambiental e as células nervosas podem representar associada mudança neural. Portanto, na constatação do DPA um dos tratamentos indicados é o treinamento auditivo, que pode ser formal ou informal, podendo ser realizado até mesmo via computador ${ }^{18}$.

A estimulação/treinamento auditivo nas desordens do processamento auditivo foi descrita ${ }^{19} \mathrm{sob}$ a visão da neurociência. $\mathrm{Na}$ referida descrição os autores afirmam que as melhoras comportamentais observadas após a realização de treinamento auditivo não estão relacionadas com modificações nos processos auditivos periféricos, e sim com o 
sistema nervoso auditivo central. Sendo assim, a plasticidade neural é fator chave nas melhoras obtidas com a estimulação auditiva e o treinamento auditivo.

Para estes autores ${ }^{19}$, ao montar um programa de treinamento auditivo deve-se ter em mente os seguintes princípios:

1. O cérebro (sistema auditivo central e sistemas relacionados) apresenta plasticidade e pode ser alterado por estimulação acústica ou pela falta dela. Os tipos de modificação incluem alterações neuroquímicas, fisiológicas e neurais. Desta forma, o TA pode melhorar o desempenho auditivo.

2. Deve-se lembrar que a plasticidade ainda está presente no sistema auditivo adulto.

3. O TA deve ser indicado a partir de resultados em testes específicos e pela história clínica do paciente.

4. A dificuldade da tarefa deve ser monitorada, de modo a ser desafiante, mas não muito difícil para não desmotivar o paciente.

5. As tarefas devem ser sistemáticas e apresentadas em grau crescente de complexidade.

6. O TA deve ser intenso, tanto no programa como um todo, como dentro da própria sessão.

Atualmente o treinamento auditivo formal (TAF) é aplicado à desordem do processamento auditivo e tem o cérebro como principal local de estimulação, o cérebro, diferentemente da audição periférica, é plástico. A melhora na função auditiva central relaciona-se com a capacidade do SNC em sofrer modificações. Já existem evidências de que o cérebro tem capacidade de adaptação em resposta ao TAF mesmo em idades mais avançadas e após longos períodos de privação. Se as áreas auditivas do cérebro anteriormente em privação podem ser estimuladas, o grau de sucesso desta abordagem de reabilitação dependerá da qualidade e da duração do acompanhamento. Alguns autores ${ }^{17}$ atribuíram grande importância à motivação do paciente para o bom resultado da estimulação, afirmando que o esforço e a motivação do paciente para realizar as tarefas são elementos chave para maximizar a possibilidade de plasticidade. Por isso, sugeriram que a mesma seja garantida por meio de reforço positivo e tarefas desafiadoras.

Um dos objetivos finais do treinamento auditivo formal deve ser melhorar o processamento de fala ${ }^{20}$, além disso, foi citado na mesma literatura como características gerais de qualquer programa de TAF: utilizar diferentes estímulos para treinar os processos auditivos, as tarefas devem ser apresentadas sistematicamente e serem gradualmente mais complexas, por exemplo, deve-se iniciar com atividades de detecção e discriminação para posteriormente partir para atividades que envolvam identificação, reconhecimento e produção, e apesar de não haver um consenso, sempre que possível - TAF deve ser intensivo com múltiplas sessões relativamente curtas durante a semana (entre 5 e 7 sessões com duração de 15 a 30 minutos).

Também é referido na literatura ${ }^{15}$ que a sequência do tratamento depende muito do tipo da atitude do paciente e caracterizaram as atitudes em quatro tipos: Tipos I, II, III e IV. O paciente com atitude fortemente positiva para a reabilitação audiológica seria o tipo I. Os fatores auditivos e psicossociais são passíveis de soluções terapêuticas e existe a aceitação do problema; o tipo II é aquele paciente com atitudes positivas, há aceitação da reabilitação, porém com algumas complicações. Pacientes do tipo III são aqueles que têm atitudes negativas, mas com um mínimo de intenção cooperativa. E, finalmente, os pacientes do tipo IV são aqueles que rejeitam o processo terapêutico.

$O$ fato de que um certo grau de neuroplasticidade se mantém durante toda a vida é descrito por alguns autores ${ }^{21}$, baseando-se neste fato, os mesmos autores afirmaram que o TAF em pacientes adultos e idosos não só provoca alterações comportamentais como também estas modificações são acompanhadas por alterações nas respostas neurofisiológicas. Recomendou-se que para um programa de TAF ser bem sucedido deve ser baseado em um diagnóstico preciso, ser realizado de forma frequente com atividades intensas e desafiadoras, ser capaz de manter a motivação do paciente e envolver orientação aos pacientes e aos familiares.

A plasticidade neural pode ser estimulada por aprendizado/treinamento ${ }^{22}$. Além disso, deve-se ensinar estratégias de audição e comunicação a portadores de deficiência auditiva, de modo a provocar alteração das associações corticais. Como características gerais do treinamento auditivo elencou-se: utilização de materiais variados, integração entre TA e estratégias de comunicação, atividades organizadas em grau crescente de dificuldade, participação ativa do paciente, viabilidade do ponto de vista custo-benefício, acessibilidade (realizado em casa), registro preciso do desempenho e reforço imediato.

O TA é um conjunto de estratégias utilizadas para desenvolver ou reabilitar as habilidades auditivas, as quais são necessárias para a compreensão da fala. Envolve estimulação da função auditiva bem como orientação a terapeutas, familiares e educadores ${ }^{16}$.

A estimulação das habilidades auditivas que deve ocorrer no TA é fundamentada no princípio da neuroplasticidade, ou seja, na possibilidade que o cérebro tem de criar novas conexões em respostas 
às solicitações a que é submetido. A repetição de uma mesma tarefa favorece o aumento do número de sinapses presentes nos circuitos neuronais envolvidos, indicando uma correlação neurofisiológica com o aprendizado. Além de auxiliar o desenvolvimento de habilidades em quem tem diagnosticado algum distúrbio no processamento auditivo, tais atividades podem melhorar a competência em processar os sons, independentemente da faixa etária. Como em quase tudo na vida, o treino leva a excelência ${ }^{10}$.

Atualmente 0 processamento auditivo vem atraindo pesquisadores de diversas áreas, incluso da Fonoaudiologia. Este enfoque multidisciplinar mostra a abrangência do processamento auditivo sobre o desenvolvimento e a manutenção da comunicação e seus distúrbios. Qualquer desajuste que dificulte e/ou impeça de tornar o sinal auditivo funcionalmente útil é caracterizado como uma desordem no processamento auditivo.

Por tratar-se de uma desordem auditiva de graves consequências para o idoso e a família, e ainda por apresentar elevada incidência em idosos acima de 60 anos, as desordens do processamento auditivo causadas pelo envelhecimento tem sido objeto de preocupações e estudos na busca de alternativas que possam minimizar seus efeitos deletérios sobre o desenvolvimento social, emocional e cognitivo do indivíduo.

As desordens auditivas são invisíveis até que seus efeitos traduzam-se no comprometimento da comunicação, ou seja, quando os indivíduos - adultos ou idosos - não conseguem mais compreender a fala, principalmente em ambientes ruidosos.

Atualmente os planejamentos terapêuticos baseados no treinamento auditivo são os mais aceitos e utilizados pelo profissional fonoaudiólogo devido aos bons resultados obtidos.

Entretanto, a falta de informação da população idosa sobre a importância da detecção precoce dessa desordem auditiva bem como a reduzida participação dos outros profissionais da área da saúde, na valorização do processamento auditivo, são possivelmente fatores importantes na detecção tardia.

Programas e campanhas envolvendo informação do que é, quando ocorre e as principais intervenções terapêuticas da desordem do processamento auditivo bem como a conscientização dos profissionais e da população em geral deve ser implementada e motivada para que se alcance uma longevidade com mais qualidade.

\section{CONCLUSÃO}

A literatura pesquisada aponta graves implicações das desordens do processamento auditivo para a comunicação do idoso. A compreensão da fisiopatologia do envelhecimento, em especial do envelhecimento auditivo, leva o ser humano a ser capaz de entender e assim contribuir para que adequadas medidas intervencionistas sejam aplicadas e dessa forma garantir a reabilitação a tempo de minimizar os efeitos dessa desordem auditiva sobre esta população.

\section{ABSTRACT \\ Background: auditory processing in elderly. Purpose: to promote a theoretical approach on auditory processing in elderly people, the disorders caused by hearing aging, as well as the resources to minimize the auditory aging impairment of the hearing abilities involved in the auditory processing. Conclusion: the alterations caused by auditory processing disorder in elderly people are many. It is necessary to continue researching in this field in order to apply adequate interventionist measures, in order to assure the rehabilitation of the individual in time to minimize the effects of the hearing disorder.}

KEYWORDS: Hearing; Aged; Auditory Perception; Acoustic Stimulation

\section{REFERÊNCIAS}

1. Quintero SM, Marotta RMB, Marone SAM. Avaliação do processamento auditivo de indivíduos idosos com e sem presbiacusia por meio do teste de reconhecimento de dissílabos em tarefa dicótica SSW. Rev Bras Otorrinolaringol. 2002; 68(1):28-33.
2. Veras RP, Mattos LC. Audiologia do envelhecimento: revisão da literatura e perspectivas atuais. Rev Bras Otorrinolaringol. 2007; 73(1):128-34.

3. Versfeld NJ, Dreschler WA. The relationship between the intelligibility of time-compressed speech and speech in noise in young and elderly listeners. J Acoust Soc Am. 2002; 111(1):401-8. 
4. Guida HL, Feniman MR, Zanchetta S, Ferrari C, Giacheti CM, Zorzetto NL. Revisão anatômica e fisiológica do processamento auditivo. Acta ORL. 2007; 25(3):177-81.

5. Samelli AG, Schochat E. The gaps-in-noise test: gap detection thresholds in normal-hearing young adults. Int J Audiol. 2008; 47(5):238-45.

6. Kozlowski L, Wiemes GMR, Magni C, Silva ALG. A efetividade do treinamento auditivo na desordem do processamento auditivo central: estudo de caso. Rev Bras Otorrinolaringol. 2004; 70(3):427-32.

7. Momensohn-Santos TM; Branco-Barreiro FCA. Avaliação e intervenção fonoaudiológica no transtorno de processamento auditivo. In: Ferreira LP, Befi-Lopes OP, Limongi SCO. Tratado de fonoaudiologia. São Paulo: Roca; 2004. p. 553-68.

8. Sanchez ML, Alvarez AMMA. Processamento auditivo central: avaliação. In: Costa SS, Cruz OLM, Oliveira JAA. Otorrinolaringologia: princípios e práticas. São Paulo: Artmed; 2006. p.191-202.

9. Ramos BD, Alvarez AM, Sanchez ML. Neuroaudiologia e processamento auditivo: novos paradigmas. RBM/ORL. 2007; 2(2):51-8.

10. Gielow I. Escutação: treino auditivo para a vida. São Paulo: Thot; 2008.

11. Pedalini MEB, Liberman PHP, Pirama S, Jacob Filho W, Câmara J, Miniti A. Análise do perfil audiológico de idosos através de testes de função auditiva periférica e central. Rev Bras Otorrinolaringol. 1997; 63(5):489-96.

12. Calais LL, Russo ICP, Borges ACLC. Desempenho de idosos em um teste de fala na presença de ruído. Pró-Fono. 2008; 20(3):147-52.

13. Strouse A, Ashmead DH, Ohde RN, Grantham DW. Temporal processing in the aging auditory system. J Acoust Soc Am. 1998; 104(4):2385-99.
14. Ferreira DR, Silva AA. Envelhecimento e qualidade de vida: a visão otorrinolaringológica. Rev Bras Otorrinolaringol. 2003; 69(Supl5):3-21.

15. Russo ICP, Almeida K, Freire CGM. Seleção e adaptação da prótese auditiva para o idoso. In: Almeida K, lório MCM. Próteses auditivas. 2. ed. São Paulo: Lovise; 2003. p. 385-407.

16. Zalcman TE, Schochat E. A eficácia do treinamento auditivo formal em indivíduos com transtorno do processamento auditivo. Rev Soc Bras Fonoaudiol. 2007; 12(4):310-4.

17. Musiek F, Shinn J, Hare C. Plasticity, auditory training, and auditory processing disorders. Semin Hear. 2002; 23(4):264-75.

18. Thibodeau LM. Computer-based auditory training (CBAT) for (Central) auditory processing disorders. In: Chermak GD, Musiek FE. Handbook of (central) auditory processing disorder: comprehensive intervention. San Diego: Plural Publishing; 2007.

19. Musiek FE, Berge BE. A neuroscience view of auditory training/stimulation and central auditory processing disorders. In: Masters MG, Stecker NA, Katz J. Central auditory processing disorders: mostly management. Boston: Allyn and Bacon; 1998. p.15-32.

20. Chermak GD, Musiek FE. Auditory training: principles and approaches for remediation and managing auditory processing disorders. Semin Hear. 2002; 23(4):297-308.

21. Bellis TJ. Deficit-specific intervention for auditory processing disorders. In: Gil D. Treinamento auditivo formal em adultos com deficiência auditiva [tese]. São Paulo (SP): Universidade Federal de São Paulo; 2006.

22. Sweetow R, Palmer CV. Efficacy of individual auditory training in adults: a systematic review of the evidence. J Am Acad Audiol. 2005; 16(7):494-504.

DOI: 10.1590/S1516-18462009005000066

RECEBIDO EM: 18/05/2008

ACEITO EM: 09/10/2009

Endereço para correspondência:

Leonardo Henrique Buss

Av. Presidente Vargas, 539

Santa Maria - RS

CEP: 97020-001

E-mail: Ihbuss@yahoo.com.br 